\title{
A Study of Lepromin in Healthy Contacts
}

\author{
DR R. N. BASU, м.в.в.s. \\ Medical Officer-in-Charge, Gandhi Memorial Leprosy Foundation Control Unit, \\ Chilakalapalle, South India, \\ DR S. R. GANGULY, м.в.в.s. \\ Medical Officer, GMLF Control Unit, Chilakalapalle, South India
}

The Lepromin test is the only test in leprosy patients which expresses the immunity and diagnosis of the patient. It has been widely used by leprologists, but comparatively less has been done to study the effects of lepromin on healthy contacts. This paper reports an attempt of a study of this nature.

\section{Selection of patients}

The Control Unit in Chilakalapalle has a training centre attached. We selected some of the trainees who came from highly endemic areas.

\section{Actual procedure}

I $\mathrm{ml}$ of refined lepromin was injected intradermally in the ventral surface of the forearm, using a sterilised tuberculin syringe and changing the needle after every injection. First reaction was usually a raised, red indurated area of $10 \mathrm{~mm}$ diameter in 24 to 48 hours. This indurated area began to disappear from the fourth day onwards and resolved completely. The site became slightly painful and tender again after two weeks and a firm nodule appeared which was also slightly painful and tender in most patients. In some patients the nodules burst and freed a discharge and the nodule in a few patients took more than two weeks to heal. The discharge was stained by carbol fuchsin in a few patients and examined microscopically. The following notes give the results of 25 patients:

I. Male 27 years - size of early reaction $50 \mathrm{~mm}$; the late reaction consisted of a nodule of $5 \mathrm{~mm}$ in diameter which burst producing an ulcer which liberated cheesy material which healed in three weeks. The patient had been working as an assistant in a leprosy hospital for the last five years.

2. Male 25 years - the early reaction was $24 \mathrm{~mm}$ in diameter; the late reaction was a nodule of $4 \mathrm{~mm}$ in diameter which became an ulcer and which healed in nearly three weeks. 'The patient had been working as an assistant in a leprosy hospital for nearly six years.

3. Male aged 25 years - early reaction was a nodule of $32 \mathrm{~mm}$ diameter; the late reaction was an indurated area succeeded by an ulcer which healed in three weeks.

4. Male aged 28 years - early reaction was a nodule of $24 \mathrm{~mm}$; the late reaction was an indurated area followed by ulcer formation which healed in three weeks.

5. Male aged 24 years - early reaction was a nodule of $4 \mathrm{I} \mathrm{mm}$; the late reaction was a nodule of $4.5 \mathrm{~mm}$ which gradually subsided. The patient had been working in a leprosy hospital for the last two years.

6. Male aged 23 years - early reaction was a nodule of $45 \mathrm{~mm}$; the late reaction was an indurated area which gradually subsided.

7. Male aged 20 years - early reaction was a nodule of $15 \mathrm{~mm}$; the late reaction was an indurated area which gradually subsided.

8. Male aged 27 years - early reaction was a nodule of $25 \mathrm{~mm}$; the late reaction was a nodule formation of $4 \mathrm{~mm}$ which healed without ulcer formation. The patient had been working as a peon (messenger) in the unit for the last nine years.

9. Male aged 45 years - early reaction was a nodule of $28 \mathrm{~mm}$; late reaction was a nodule of $4 \mathrm{~mm}$ which healed smoothly. The patient had been working as a peon in the unit for the last I I years.

I0. Male aged 24 years - the early reaction was a nodule of $28 \mathrm{~mm}$; the late reaction was an indurated area which gradually subsided. The patient is on the staff of the unit.

I I. Male aged 20 years - the early reaction was a nodule of $20 \mathrm{~mm}$; the late reaction was an indurated area which gradually subsided. The patient was watchman of the Unit for three years. 
I 2. Female aged 29 years - the early reaction was a nodule of $14 \mathrm{~mm}$; the late reaction was an indurated area which gradually subsided. The patient was on the staff of the unit for the last three years.

13. Male aged 20 years - the early reaction was a nodule of $18 \mathrm{~mm}$; the late reaction was an indurated area of $50 \mathrm{~mm}$. The patient worked for one year in the Unit.

I4. Male aged 22 years - the early reaction was a nodule of $14 \mathrm{~mm}$; the late reaction was an indurated area of $13 \mathrm{~mm}$. The patient worked for three years.

I 5. Male aged 2 I years - the early reaction was a nodule of $12 \mathrm{~mm}$; the late reaction was an indurated area of $10 \mathrm{~mm}$. The patient worked for one year.

i6. Male aged 2 I years - the early reaction was a nodule of $12 \mathrm{~mm}$; the late reaction was an indurated area of $10 \mathrm{~mm}$. Still working in the Unit.

I 7. Male aged I9 years - the early reaction was a nodule of $16 \mathrm{~mm}$; the late reaction was an indurated area of $9 \mathrm{~mm}$.

I8. Female aged 22 years - the early reaction was a nodule of $16 \mathrm{~mm}$; the late reaction was an indurated area of $10 \mathrm{~mm}$.

I9. Male aged 20 years - the early reaction was a nodule of $\mathrm{I} 0 \mathrm{~mm}$; the late reaction was an indurated area of $8 \mathrm{~mm}$.

20. Male aged i9 years - the early reaction was a nodule of $10 \mathrm{~mm}$; the late reaction was an indurated area of $8 \mathrm{~mm}$.

21. Male aged 22 years - the early reaction was a nodule of $19 \mathrm{~mm}$; the late reaction was an indurated area of $6 \mathrm{~mm}$.

22. Male aged 22 years - the early reaction was a nodule of $10 \mathrm{~mm}$; the late reaction was an indurated area of $6 \mathrm{~mm}$.

23. Male aged 27 years - the early reaction was a nodule of I $\mathrm{Imm}$; the late reaction was an indurated area of $5 \mathrm{~mm}$.
24. Male aged 22 years - the early reaction was a nodule of $19 \mathrm{~mm}$; the late reaction was an indurated area of $8 \mathrm{~mm}$.

25. Male aged 2 I years - the early reaction was a nodule of $14 \mathrm{~mm}$; the late reaction was an indurated area of $\mathrm{I} 0 \mathrm{~mm}$.

\section{S U M M A R Y}

Some 30 paramedical workers and staff workers of Chilakalapalle Control Unit (originating from different parts of India), who came for leprosy training were selected and took part in an experiment to study the results of the lepromin test in healthy contacts. It was found that all subjects reacted positively and in some the late reaction was pronounced and an ulcer developed on this site of inoculation.

\section{DISCUSSION}

It is now well known that most healthy contacts of leprosy suffer from repeated subclinical infection and by means of it acquire immunity against leprosy. The contact of the above subjects with a leprosy patient from time to time had apparently allowed a small number of leprosy bacilli to enter the body and so stimulate the histiocytes to minimum control of the vascular and to the limitation of the infection. The experiences seem to have stimulated the defence mechanism so that it reached its peak at the age of 20 years. The resistance seems more evident in those who work in the field of leprosy for some years, perhaps because they come into more intimate contact with leprosy patients in such a way that greater degree of immunity can evolve. It follows that carrying out lepromin testing in endemic areas will assist in detecting those who have not acquired immunity against leprosy and enable precautions to be taken against any dangerous degree of contact. The lepromin test itself is, of course, valuable in the prevention of leprosy when carried out with discretion and ingenuity. 